Short notes and reviews

\title{
Of cavities - and kings
}

\author{
Frederick R. Schram \\ Institute for Systematics and Population Biology, University of Amsterdam, P.O. Box 94766, \\ 1090 GT Amsterdam, The Netherlands
}

Keywords: phylogeny, coelom, pseudocoelom, invertebrates

Review of and commentary on: Body Cavities: Function and Phylogeny, edited by G. Lanzavecchia, R. Valvassori, and M.D. Candia Carnevali. Mucchi Editore/Modena, 1995, 280 pp., ISBN 88-7000-259-4.

"The time has come", the Walrus said,

"To talk of many things:

Of shoes - and ships - and sealing wax-

Of cabbages - and kings -

And why the sea is boilling hot -

And whether pigs have wings."

Louis Carroll

I must confess this passage from Carroll has, until now, never made much sense to me, which is to say it is merely nonsense - but only maybe. We might wonder if Louis Carroll's famous walrus was not in fact a phylogeneticist? The walrus certainly had a wide array of characters under consideration, was aware of the role of "higher authority", metaphorically alluded to the intellectual ferment of considering so many disparate features, and even managed to pose a phylogenetic hypothesis at the end. What the walrus lacked only was method. Which brings us to this book. We have a considerable array of features and taxa reviewed herein, there are constant references to "differing opinions of authority", and naturally any such disagreement among scientists will always keep things boiling. So far so good. However, what the volume seems to have, as opposed to the famous walrus, is a disinclination for its authors to entertain hypotheses.

Phylogeny of the animal kingdom has become an area of active concern again after decades of dormancy. This book is another in a mounting body of contemporary literature that will figure in this regard. In addition, the interest in the use of rigorous techniques of analysis of the phylogenetic relationships of the animal phyla has come into its own. This book, unfortunately, for the most part lies outside of this latter sphere. This is not to say there is not much of interest in this volume, but I suspect that the reader will be more than a little disappointed that in the end so little seemed to be achieved.

This is a collection of papers delivered at one of the periodic symposia of the Unione Zoologica Italiana. The participants were mostly Italian scientists, but there was an inclusion of participants from other countries as well. The idea behind the symposia is " "...to throw some light on a problem that may be new or old, but always of current interest." (the editors: p. 7). In that respect, the organizers and editors did a good job. It is their participants who did not always cooperate and rise to the occasion.

The chapters of this book can be organized into three major categories: phylogenetics, development, and function and anatomy. However, this division is not easily evident from the framework of the book since chapters within these three fields are scattered throughout the volume. The phylogeny papers generally occur early in the sequence of articles, and function and development tend to occur later. Most of the contributions are what can be termed full-fledged papers. However, there are writings that obviously are 
only summaries of what was actually presented at the symposium, and one of these is little more than an abstract. Most of the articles are well illustrated, but other very hefty contributions have only text and lack any illustrations to assist the reader in following arguments. Most of the papers contain a lot of information, but several of these lack any summary or conclusions; they just end and thus make no attempt to try and place what had preceded the paper's reference lists in any kind of broader context. Therefore, I am afraid that readers and users of this book are going to find that in some cases it is rather difficult and frustrating to try and follow its contents.

Lamentably, I noted a tendency in all too many of the papers to utilize "jargon". For example, I was never really sure just what "organogenetic components" were, although one author (Ghiara, p. 212) seemed to equate these with the old and well-established concept of presumptive cells or tissues. In other instances, however, I was not sure if in fact some of the author(s) had something else in mind - maybe they were using some kind of "new-speak" reference to genetic activation (1?). It seems that the more English becomes the international language of science, the more determined users seem to become in making it a lingua obscura.

Let us survey the chapters in the three fields mentioned above. The first group of papers deals with phylogeny.

Alberto Simonetta and Emilio Insom have a general paper that incorporates information from fossils and pays close attention to the history of the various issues they take up. The authors admit that "... the examples [they treat] ... have little in common ...", which in fact is a bit exasperating, stretching as they do from brachiopods, through articulates, to chordates. Nevertheless, Simonetta and Insom come up with some generally valid conclusions. The first is that to neglect consideration of classical morphology in phylogenetic analysis is a serious error. The authors do not explicitly state it, but the problems with morphology are not problems of lack of information, but rather problems of insufficiently rigorous analysis. Second, scientists who wish to take up the issues of evolution should be conversant with all lines of evidence, fossil as well as modern forms, morphology and well as molecules, because no line of information "should be privileged above others". In fact, many of the later chapters in this book quite ignore this warning and continue to suggest that single sources of information (molecules, ultrastructure, whatever) will hold the key to resolving phylogenetic problems. Third, commentators on phylogenetic issues should take a "holistic, morpho-functional approach." This is grand sounding, but again I think it is an instance, as mentioned above, of inflated jargon acting to obscure just whatever it is the authors intended to convey. If they seek to reinforce their first and second dicta, then so much the better. Fourth, workers in animal phylogeny should not take up the dichotomous keys developed for identifying species as a tool for the development of phylogenies. This is a critical issue. All too often there is a complete lack of phylogenetic perspective on the part of alpha-taxonomists in their work. Just how this can be so I have never understood, but it is an established fact. So to try and employ a product of an alpha-taxonomist directly into phylogenetic analysis is often impossible; one has to go to the animals anew and work from first principles.

Pat Willmer puts forth a chapter on "modern approaches". However, there is in fact nothing particularly modern about the old evolutionary systematic methods she espouses, and much of what she writes is elaborated on more completely in Moore \& Willmer (1997). Willmer's thesis is that cladistic methods "have not resolved the situation" as far as phylogenetic relationships of the phyla and that the results derived from molecules are "inconclusive". She apparently chooses to ignore the real resolutions that have emerged from the cladistic analyses of people like Nielsen et al. (1996), but then Willmer prefers a method that focuses on just a few body features when dealing with phylogenetic issues. What she is doing here is putting the scenario, which might explain aspects of animal evolution, before the actual hard analysis of available data. Consequently, in the tradition of Sydney Manton she sees "multiple origins" everywhere, and she prefers to focus on the differences between phyla 
rather than on their shared similarities. She tells us that cladistic methods. fail because they get what they want "according to preconceptions of what to put in" the analysis. This is manifestly not true! As a concrete example, just examine the trees of Nielsen et al. (1996) derived as they are from the application of rigorous cladistic techniques, and which stand at odds with what Nielsen (1995) thought they "should" be. Cladistics need not, indeed should not, be done with preconceptions, while the method that Willmer espouses always is.

Three contributions deal with specific groups or clusters of groups. Reinhardt Kristensen has an enticing titbit on pseudocoelomates and "new" yet to be described taxa from Greenland. Unfortunately it is only an abstract. Elvezio Ghirardelli includes a paper on Chaetognatha and the "unresolved problems." He seems to have the elements of two separate articles here, offering comments on the one hand on the structure and development of the body cavity in arrow worms with a view towards their being somehow within the deuterostomes, and on the other hand favorably commenting on the conclusions of others, especially Telford \& Holland (1993), that suggest chaetognaths are an early offshoot of the metazoan lineage. In the end, Ghirardelli puts his faith in molecules to eventually solve the problems here, but in doing so he completely ignores some of the specific hypotheses that analysis of morphology has already come up with (e.g., see Meglitsch \& Schram, 1991; Nielsen, 1995; Schram \& Ellis, 1995; Nielsen et al., 1996). Perhaps this is so since, with the exception of some references to relatively recent papers that sort of "fine tune" anatomical knowledge in the group, the key reference work on chaetognath anatomy for Ghirardelli still remains Grassi (1883).

The third of these papers is by Robert Wallace and colleagues. This is without doubt the best and most stimulating piece of science in the book. This team was responding to the challenge of Clark (1964) that claimed because there is a conflict of opinions among various authorities that it was impossible to establish "a coherent picture" of the evolution of pseudocoelomates. Nevertheless, in a short, very concise paper, these authors take up consideration of three data bases: one derived from Willmer (1990), another from Meglitsch \& Schram (1991), and one of their own. The Willmer data yielded too many trees to be useful. The trees from Schram's data had some interesting variants depending on whether one included chaetognaths (6 equally parsimonious trees) or deleted that phylum (a single tree). Their own data yielded a single, though different, tree. As to which of these two trees might be the better, is irrelevant. What is important is that there is now a clear set of hypotheses about the phylogenetic relationships within this cluster of taxa.

Moreover, the authors further demonstrate the rigor of the cladistic method. There have been suggestions (e.g., Ruppert, 1991) that pseudocoels and cuticular structures should no longer be used ir phylogenetic analyses of aschelminth groups since the chances of "convergence" in these features are too high. In response, Wallace et al. take their data bases and delete the offending features. They then re-ran the analyses and got exactly the same trees! The assertions of Ruppert, in short, prove to be irrelevant. This demonstrates clearly that when one tries to focus on a few characters and extrapolate phylogenies from them, one skates on very thin ice. It is the total amount of available information for a set of taxa that determines their phylogenetic relationships. In another way, this disproves again that Willmer's contention that it is the preconceptions that upset the analysis is simply not true. Hypotheses are hypotheses, and they can be subjected to a test either by deleting data or adding new data and noting the effects. To do this is science; to speculate on a single or a few characters is to weave a just-so story that Carroll's walrus might enjoy.

Finally, Alessandro Minelli offers some comments relevant to the problems of assessing homology and making phylogenies. Minelli too mentions the great plethora of opinions that prevail among "authorities" when pronouncing on phylogenetic questions. However, rather than wringing his hands and bewailing that we are thus unable to conclude anything, he simply asks what do we need to know about coeloms and cavity formation to make this feature more useful in a 
phylogenetic context. It is sound advice, don't "pass the buck", just do the work!

The second grouping of chapters in this book deals with development, but to my surprise only two papers really take these issues up. Eduardo Boncinelli and co-workers examine the expression of homeobox genes in vertebrate brains. It is an interesting paper but has really little to do per $s e$ with the focus of the book.

Gianfranco Ghiara has a major paper on reviewing the history of the study of early vertebrates and their gastrulation. There is indeed quite an historical catalogue in this contribution. He makes a convincing case, however, for getting rid of what he believes is an outdated and, indeed, intellectually constraining, body of germ layer theory. Ghiara would prefer to replace the traditional theory of three germ layers with one that entails consideration of a series of presumptive areas or cell clusters: in his listing epiblasts, neuroblasts, chordoblasts, mesoblasts, and hyperblasts. A drawback of this contribution though is that he does not get into the developmental genetic issues here until the end of the paper and then only in a summary manner. It would have been interesting to see how the Ghiara concept of developmental programming could be tied into specific examples of gene action and control. Perhaps this will come.

The last group of papers in the volume constitutes the largest section. In many respects, I find these the least satisfactory. Gianni Amirante and associates write about immune systems in arthropods. Nicplo Parinello also discusses immune functions of coelomic fluid in echinoderms. Giuseppe Scapigliati and colleagues focus on stick insect hemocytes. Giorgio Bavestrello heads a group that examined canal systems in demosponges. Fascinating and interesting as these papers were, and despite what has obviously entailed a considerable amount of careful and detailed research, the disharmony of these contributions with the title of the book jarred me. I kept asking myself repeatedly as I went through these papers, "But what does this have to do with the function and phylogeny of body cavities?"

Robert McNeill Alexander does a more credible job in this respect, although most of what he presented in his review of hydrodynamic mechanisms had essentially been published earlier and elsewhere. Not all the papers in this group seemed to lack focus, but that does not mean that they did not raise other problems.

Condia Carnevali and her associates present a detailed examination of the lantern coelom in echinoids and its "morpho-functional adaptation." The tissues here form an extremely complex structure. The research team offers a marvelously detailed ultrastructural study, while again making allusions to "organogenetic components." However, their conclusions on page 161 disappoint in that they are really generic, all purpose generalities that do not really offer us much to sink our teeth into.

Giulio Lanzavecchia heads a group that examines body cavities in nematomorphs. The contribution is really too short and lacks an abstract. It contains some interesting new information on nematomorph cavities associated with gonads, which have hydrostatic as well as gonadal functions. However, they literally leave us dangling with regard to the supposed apomorphies that nematomorphs share with nematodes and that they list early in the paper (p. 45): viz.., longitudinal body wall muscles, dorso-ventral muscle flexion, and adult cuticle similarities. I would have liked to have seen here some indications that this group of researchers had in fact come in contact with the group under Wallace (reviewed above), and that the latter group had in fact things to say to this team about techniques of analysis which indeed they do but I see no evidence here of any effect.

Luitfried von Salvini-Plawen and Thomas Bartolomaeus focus on the histology of the gonopericardium in molluscs. They find that in molluscs these tissues differentiate separately from the myoblastoma and are not an integral part of that area, as is the case in the "true" coelomates. They make some interesting comparisons of their findings to situations in the pseudocoelomates, especially nematodes, but surprisingly not to the nemertines, who put their "coelomic blood vessels" to similar uses. The big problem with their contribution in my mind is that they again focus on the results from consideration of one system 
and from this narrow base then begin to exclude phylogenetic possibilities. Certainly the results of the Wallace group vis-à-vis the suggestions of Ruppert should have been a warning against doing this sort of thing.

Finally, Ulrich Welsh discusses the histo-morphology of deuterostome coeloms. He presents some considerable ultrastructural detail. However, he never came to any meaningful conclusions about "evolution" despite the title of his paper. His contribution just sort of ends, and we are left hanging as to the larger scale significance of his work.

Well, what are we left with from all of this? Some disturbing things emerge. I may be totally off base here, but I think a lot of what is in this book is a reflection of what is all too rampant in the field of zoology today. I get the distinct impression that, despite wonderfully careful and high quality technical work being done in laboratories across the world, in many cases a true evolutionary perspective is totally lacking. We in biology always raise the mantra that evolution is the core framework of all we do. Now in medical research this unifying principle can understandably shift to the background in deference to more applied issues. However, in a book about "function and phylogeny," the evolutionary principle should have been foremost. Are we as scientists becoming so complacent about this integrative axiom that we feel it need not be employed in a forceful and forthright manner? I hope not, because I fear that a science that becomes satisfied with just recording knowledge for knowledge's sake - the tireless, unending examination of minutiae - divorced from larger scale theoretical issues is a science in decline.

In the past several years, a lot of very good, stimulating, and synthetic phylogenetic work on the phylogeny of phyla has been published (e.g., Ax, 1995; Brusca \& Brusca, 1990; Eernisse et al., 1992; Meglitsch \& Schram, 1991; Nielsen, 1995; Nielsen et al., 1996; Schram, 1991; Schram \& Ellis, 1995). The results of these studies do not always agree in details, but certain broad scale patterns appear in each of these works. For example, I could offer some consensus trees derived from my own work on the subject (Fig. 1). It is clear that even with the limited data base this is built on (see Schram \& Ellis, 1995) that some very clear hypotheses of relationships of phyla emerge from this work and which are relevant to issues dealt with in this book. For example, Chaetognatha bear a clear relationship to pseudocoelomate phyla. The nematomorph/nematode link is not as strong as one might have suspected. There appears to be a transition series of "filter feeding" phyla initially focused around sessile lophophorate types with tri-partite coeloms and leading to more mobile phyla that include the chordates. Spiral cleavage would seem to be a more important factor in organizing a "protostome" clade than coelom formation. Mollusca would appear to be securely nested within a clade wherein coelom formation is focused on the development of the myoblastema. These trees, however, are not the final say in the matter. For example, despite what the majority rule tree shows, there is insufficient information $(66.6 \%$ arbitrary cut-off point) in the data base at present to adequately sort the arthropod and near-arthropod groups. There are clearly also problems involved near the base of the "coelomate" clade, and from inspection of the character optimization this is linked in large part to the status of the entoprocts. However, data bases are not frozen in time. In fact, we at the Institute for Systematics and Population Biology are in the processes of linking our morphological studies, directed at building a much expanded and more inclusive data base, with a team of developmental geneticists focused on study of certain aspects of spiral cleavage and control of mesoderm formation. Combining our respective data bases within a cladistic framework can only raise the levels of certainty about some of these phylogenetic branches.

No matter what new things we may discover about animal morphology, development, or molecules in the future it, nevertheless, seems clear that the resultant robustness of the synthetic, to say nothing of cladistic, approach can no longer be doubted. Therefore, it becomes doubly frustrating to encounter a collection of papers like this that, in instance after instance, focuses on one or two narrow anatomical systems or parts of systems and then extrapolates grand phylogenetic patterns from that narrow base. Have we learned 

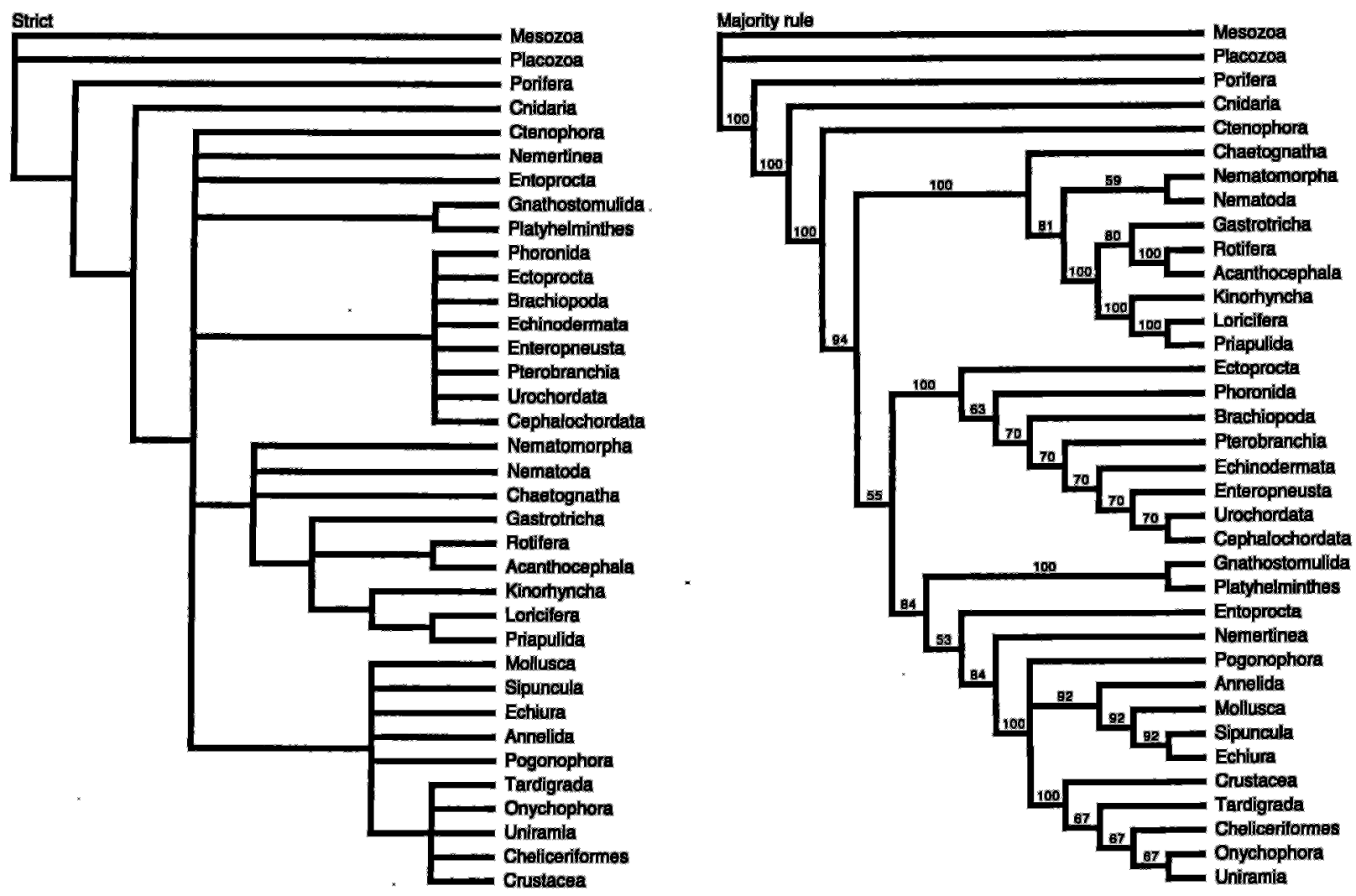

Fig. 1. Strict and 50\% Majority Rule consensus trees of data employed in Schram (1991) with some corrections for new and altered information over that used in Schram \& Ellis (1995). These trees result from some 384 equally parsimonious trees of length 124 , consistency index of 0.50 , retention index of 0.74 , and rescaled consistency index of 0.37 . Even with such an admittedly limited data set a great deal of structure is evident from the strict consensus. The sequence of "lower" phyla is clear, and certain clades within the "higher" are well established: viz. acoelomates, lophophorate/deuterostomes, pseudocoelomates, and "higher" protostomes. The $50 \%$ majority rule consensus reveals the most likely pattern derivable from these data. However, the low values in the center of the tree, viz., concerning the position of the Entoprocta, the relationship of the "spiralian" to the lophophorate/ deuterostomes, the base of the lophophorate clade, and the relationships within certain members of the pseudocoelomates, are correlated with ambiguous or missing information concerning the nature of spiral cleavage, control of mesoderm formation, and unresolved aspects of internal soft anatomy of many of these groups. Nevertheless, these trees summarize a series of hypotheses of relationships among these taxa and place some assessment of the strength or weakness of certain branches.

nothing in the last decade?! Or do we prefer merely to participate in conferences at nice venues, with congenial colleagues, good 'food and wine, and an outlook that says: "God forbid, that we should ever disagree in such pleasant circumstance", - or learn new, different, and sometimes painful ways to re-examine our own assumptions based on the proclamations of "recognized authorities." Science under the best of circumstances should not be so conservative a monarchy, but rather a very vibrant and dynamic democracy.

I think that the crisis here is not merely one of conflicting methods. It grows out of a more fundamental dialectic in evolutionary science. There exists a real split between researchers who see their science in an idealist manner, and those who operate as realists (Schram, 1993). The idealist viewpoint forces its adherents to take up reductionist positions in seeking knowledge. Experiments, manipulation of parts, and a heart-felt commitment to rigid cause-and-effect reasoning characterize this school. These people, whether they realize it or not (and they would probably vehemently deny it), function in an essentially Lamarckian milieu. Environmental perturbations 
and functional necessities serve as the source of traits, a source that can repeatedly and independently yield the same effects in different places, at different times, in different taxa. Structures have functions, functions satisfy purposes, purposes develop from constraints, and the constraints govern the structure - all more than a bit circular. Historical lineage is not as important as functional causes in analyzing animal history. The differences between species for these people seem to compel explanations. Although these researchers often preach holism, their philosophic viewpoint compels them to focus only on parts of whole organisms.

The realists on the other hand revel in a certain scepticism concerning experiments. They are materialists who often prefer observation to laboratory manipulation. They approach the field as synthesizers, focusing on the similarities that one can see between species. To adherents of this school (essentially Darwinian in character), historical lineage is the important element in studying and seeking to understand traits - whether these features come from morphological, molecular, or developmental investigations. "Blood is thicker than water" to these people - trying to determine familial relationship serves better as a motivating force in studying nature than manipulation of mediums of the environment.

Morphology does not have a lock on the path to enlightenment, even though I happened to be more comfortable working with morphology. Molecules have a lot to contribute as well. However, this contribution should not stand as a separate source of knowledge but should merge together with morphology for a truly synthetic and total evidence type of approach. As examples, Poe (1996), Shaffer et al. (1997), and Wheeler et al. (1993) have demonstrated the efficacy of these sorts of total evidence analyses in producing robust hypotheses of phylogenetic relationships. The techniques are there, the data are there, the knowledgeable authorities are there. So why are we still locked on methodological outlooks that should have died decades ago with Lybie Hyman and Sidnie Manton?

Finally what about all those conflicting opinions and the "imperfect" nature of the data that so many scientific workers seem to be concerned about. The solution to this is clear. We have to realize that unanimity of agreement over the perfect and complete data set is a type of scientific "holy grail." Moreover, just like that other grail, unanimity and perfection are myths! We are not in a quest for absolute truth when we study animal phylogeny, we are trying to extract the pattern that is implicit in a widely uneven, incomplete, sometimes even inaccurate, but always-capable-of-improving data base. Herbert Spencer stated it well when he said "Science is organized knowledge." However, we cannot, and should not, sit back and merely be content with pronouncing evolutionary scenarios based on a few assumptions and reigning paradigms. We already, or at least should, know that that emperor has no clothes. "However, the analysis of real data can provide at least "a partial, but nonetheless wellgrounded, foundation upon which to base hypotheses.

We must simply take all the information we have relevant to discerning phylogeny and use it. As to what results will come out of this attempt, we do not know. However, I am convinced that it does not have to be perfect. The pattern that emerges from the analysis is, above all, most important as a guide to future work and as a method for testing past assumptions and assertions. The actual trees merely help us visualize the results. We fully expect that the patterns seen in the trees like that of Fig. 1 will change as our data. base is expanded with more morphologic, new developmental, and even molecular data. A phylogenetic tree is not truth, eternal and immutable, rather it is organized information, an extraction of pattern. And that should be the name of this game.

\section{References}

Ax, P., 1995. Das System der Metazoa I: ein Lehrbuch der phylogenetischen Systematik: 1-226 (G. Fisscher Verlag, Stuttgart).

Brusca, R.C. \& G.J. Brusca, 1990. Invertebrates: i-xvilï, 1922 (Sinauer, Sunderland).

Clark, R.B., 1964. Dynamics in metazoan evolution: the origin of the coelom and segmentation: $i-x, 1-313$ (Clarendon Press, Oxford). 
Eernisse, D.J., J.S. Albert \& F.E. Anderson, 1992. Annelida and Arthropoda are not sister taxa: a phylogenetic analysis of spiralian metazoan morphology. Syst. Biol, 41: 305-330.

Grassi, G.B., 1883. I Chetognati. Fauna Flora Golf. Neapel., 5: 1-126.

Moore, J. \& P. Willmer, 1997. Convergent evolution in invertebrates. Biol. Rev., 72: 1-60.

Meglitsch, P. \& F.R. Schram, 1991. Invertebrate zoology, 3rd ed.: 1-ix, 1-623 (Oxford Univ. Press, New York).

Nielsen, C., 1995. Animal evolution: Interrelationships of the living phyla: i-ix, 1-467 (Oxford Univ. Press, Oxford).

Nielsen, C., N. Scharff \& D. Eibye-Jacobsen, 1996. Cladistic analyses of the animal kingdom. Biol. $J_{x}$ Linn. Soc., Lond, 57: 385-410.

Poe, S., 1996. Data incongruence and the phylogeny of crocodilians. Syst. Biol, 45: 393-414.

Ruppert, E.E., 1991. Introduction to the aschelminth phyla: a consideration of mesoderm, body cavities, and cuticle. In: F.W. Harrison \& E.E. Ruppert (eds.), Microscopic anatomy of the invertebrates, 4: 1-17 (John Wiley, New York).
Schram, F.R, 1991. Cladistic analysis of metazoan phyla and the placement of fossil problematica. In: A.M. Simonetta \& S. Conway Morris (eds.), The early evolution of metazoa and the significance of problematic taxa: 35-46 (Cambridge Univ. Press, Cambridge).

Schram, F.R., 1993. The British School: Calman, Cannon, and Manton and their effect on carcinology in the English speaking world. Crust. Issues, 8: 321-348.

Schram, F.R. \& W.N. Ellis, 1995. Metazoan relationships: a rebuttal. Cladistics, 10: 331-337.

Shaffer, H.B., P. Meylan \& M.L. McKnight, 1997. Tests of turtle phylogeny: molecular, morphological, and paleontological approaches. Syst. Biol., 46: 235-268.

Telford, M.Jx \& P.W.H. Holland, 1993. The phylogenetic affinities of the chaetognaths: a molecular analysis. Mol. Biol. \& Evol., 10:"660-676.

Wheeler, W.C., P. Cartwright \& C.Y. Hayashi, 1993. Arthropod phylogeny: a combined approach. Cladistics, 9: 1-39.

Willmer, $P_{n}, 1990$. Invertebrate zoology: Patterns in animal evolution: i-xiii, 1-400 (Cambridge Univ. Press, Cambridge).

Received: 5 August 1997 